\title{
Relación entre los estilos educativos parentales o prácticas de crianza y la ansiedad infanto-juvenil: una revisión bibliográfica
}

\section{The relationship between parenting styles or parenting practices, and anxiety in childhood and adolescence: a systematic review}

\begin{abstract}
Dra. Elvira MARTíNEZ BESTEIRO. Profesora Contratada Doctora. Universitat de València (elvira.martinez@uv.es). Ana JULIÁN QUINTANILLA. Investigadora independiente (ajuquin@alumni.uv.es).
\end{abstract}

\section{Resumen}

La ansiedad es una de las patologías más comunes en la población infanto-juvenil y presenta una elevada comorbilidad que en ocasiones dificulta el diagnóstico y el tratamiento pertinente. Asimismo, tiende a persistir a lo largo del tiempo y provoca grandes dificultades en la vida diaria de los niños y adolescentes. En esta revisión se ha analizado la evidencia empírica sobre la relación de los estilos o prácticas de crianza con la ansiedad en la población infanto-juvenil a partir de una revisión bibliográfica basada en veintidós artículos. Se han encontrado evidencias bastante consistentes que relacionan el afecto, la calidez y la aceptación con menores niveles 0 síntomas de ansiedad o síntomas internalizantes en población generalmente europea; y el rechazo, el control psicológico, el excesivo control, la sobreimplicación, la disciplina rígida, el control hostil y el estilo autoritario, con mayores niveles de ansiedad o síntomas. También se ha puesto de manifiesto la necesi- dad de desarrollar programas preventivos de educación familiar, e incluir las prácticas de crianza como elementos clave a trabajar en los programas de intervención y tratamiento de la ansiedad infanto-juvenil.

Descriptores: Estilos educativos, prácticas de crianza, ansiedad, infancia, adolescencia, revisión bibliográfica.

\section{Abstract}

Anxiety is one of the most common pathologies in the childhood and adolescent population and it has a high comorbidity that sometimes makes diagnosis and treatment very difficult. It also tends to persist over time, causing great difficulties in the daily life of children and adolescents. In this review the empirical evidence for the relationship between parenting styles and practices and anxiety in children and adolescents is analysed based on twenty-two articles. Rea-

Fecha de recepción de la versión definitiva de este artículo: 10-12-2016.

Cómo citar este artículo: Martínez Besteiro, E. y Julián Quintanilla, A. (2017). Relación entre los estilos educativos parentales o prácticas de crianza y la ansiedad infanto-juvenil: una revisión bibliográfica. Revista Española de Pedagogía, 75 (267), 337-351. doi: 10.22550/REP75-2-2017-10 
sonably consistent evidence was found, based mainly on European populations, that lower levels of anxiety, or fewer anxiety symptoms or internalising symptoms were related to affection, warmth, and acceptance from parents. In contrast, higher levels or more of these symptoms were linked to authoritarian style, rejection, psychological control, excessive parental control, over-involvement, rigid discipline, and hostile control. Furthermore, the review provided evidence of the need to develop preventive family education programmes and include parenting practices as an important part of the process for treating childhood and adolescent anxiety.

Keywords: Parenting, anxiety, childhood, adolescence, bibliographic review.

\section{Introducción}

La ansiedad es una problemática común en la población infanto-juvenil, presenta una alta comorbilidad, tiende a persistir a lo largo del tiempo y provoca grandes dificultades en el funcionamiento social, emocional y funcional del individuo.

Además, una de las características más relevantes de la infancia y la adolescencia es la denominada determinación ambiental, esto es, la dependencia de esta población respecto a los adultos, que les hace particularmente vulnerables a múltiples influencias que escapan de su control e influyen en su comportamiento y en el modo de afrontar situaciones específicas. Por lo tanto, los factores familiares adquieren un papel fundamental en el ajuste y desarrollo del menor. Más concretamente, se ha señalado el estilo educativo «como una de las variables que mayor influencia ejerce en el desarrollo emocional y social del niño» (Sala, 2002, p. 544).

Los estilos educativos, según Pérez y Cánovas (1996, citado en Aroca, Cánovas y Sahuquillo, 2014, p. 195) son «modelos 0 esquemas prácticos que simplifican las pautas de crianza y educación paterna en determinadas dimensiones básicas que, cruzadas entre sí en diferentes condiciones, dan lugar a diversos y habituales tipos de educación familiar». Algunos autores, como Darling y Steinberg (1993), estiman necesario diferenciar entre los estilos y las prácticas de crianza. Para estos, el estilo educativo es definido como el conjunto de actitudes, metas y patrones de crianza que crean un clima emocional en la relación padres-hijos, mientras que las prácticas de crianza constituyen formas específicas de interacción de los padres con sus hijos en situaciones concretas.

Asimismo, en las aproximaciones teóricas sobre los estilos educativos se distinguen dos enfoques: el enfoque tipológico, que clasifica a los padres según su estilo educativo, definido a partir de la relación entre varias dimensiones; y el dimensional, que trata de poner en relación alguna de las variables 0 dimensiones más relevantes del estilo educativo, con variables referidas al ajuste 0 competencia de sus hijos (Oliva y Parra, 2004). No obstante, ambos enfoques pueden considerarse complementarios (Oliva, Parra, Sánchez-Queija, López, 2007) y están muy 
relacionados, ya que «las dimensiones que los investigadores con más frecuencia han puesto en relación con el ajuste del adolescente son precisamente aquellas que sirven para constituir las tipologías de los estilos parentales» (Oliva y Parra, 2004, p. 109). Estas dimensiones son el afecto y el control. Como señalan Darling y Steinberg (1993), más allá de los modelos teóricos empleados, casi la totalidad de las investigaciones se han basado en estas dos dimensiones. Por lo tanto, la presente revisión se va a centrar en el análisis de estas dos dimensiones en aquellos estudios que adopten un enfoque dimensional.

La dimensión afecto se refiere a la calidez, el apoyo y cariño en la interacción, la capacidad de respuesta de los padres, la aceptación de los sentimientos y comportamientos de sus hijos y la participación en sus actividades. Esta dimensión aparece etiquetada en algunas investigaciones como aceptación, calidez o calor, atención, cuidado 0 apoyo. Algunos estudios también investigan el polo opuesto a esta dimensión: el rechazo, la crítica, la hostilidad o la negatividad (Bögels y Brechman-Toussaint, 2006; Luis, Varela y Moore, 2008; McLeod, Wood y Weisz, 2006; Wood, McLeod, Sigman, Hwang y Chu, 2003).

La dimensión control se define como el conjunto de límites y reglas, las regulaciones de los padres para canalizar el comportamiento de los hijos, el conocimiento de las actividades de los mismos y la exigencia de responsabilidades (Betancourt y Andrade, 2011; Oliva et al., 2007). No obstante, la mayoría de los estudios hacen referencia al control en su polo más extremo, entendiéndolo como la excesiva regulación y restricción de las actividades y conduc- tas de los menores, la implicación excesiva en la toma de decisiones de sus hijos y la limitación de la autonomía. Esta dimensión también aparece etiquetada en algunos estudios como restricción, exigencia, rigidez, sobreimplicación o disciplina. $\mathrm{Su}$ polo opuesto es la concesión o promoción de la autonomía (Aroca et al., 2014; Bögels y Brechman-Toussaint, 2006; McLeod et al., 2006; Wood et al., 2003). Asimismo, algunos autores resaltan la necesidad de establecer una diferenciación entre el control psicológico y el conductual, ya que tienen implicaciones diferentes sobre el ajuste del menor. El control conductual define las conductas de "atención y supervisión de las actividades de los hijos, referido en varias investigaciones como supervisión 0 monitoreo» (Betancourt y Andrade, 2011, p. 28). El psicológico es un tipo de control más intrusivo, coercitivo y manipulador de los pensamientos y sentimientos de los hijos, que se manifiesta a través de estrategias como la inducción de la culpa 0 chantaje afectivo, la invalidación de sentimientos y la sobreprotección (Betancourt y Andrade, 2011; Oliva et al., 2007).

Por otra parte, desde los modelos cognitivos explicativos de la ansiedad, diversos autores plantean hipótesis sobre cómo influyen ciertas dimensiones de los estilos educativos en la aparición, desarrollo y mantenimiento de la ansiedad. Así, por ejemplo, Chorpita y Barlow (1998) teorizan que el control excesivo por parte de los padres restringe al niño la capacidad de manipular y relacionarse con el medio ambiente, limitando las conductas para explorar nuevas situaciones de forma independiente. Igualmente, la falta de calidez, aceptación 0 cuidado hacen ver a los me- 
nores que no cuentan con apoyo ante las nuevas situaciones y que sus acciones no tienen influencia sobre el entorno, de forma que pueden desarrollar un sentimiento de impotencia. Ambas prácticas pueden transmitirles una percepción del entorno como amenazante sobre el cual no tienen control. En la línea de estos autores, Dadds y Barret (2001), Hudson y Rapee (2001) y Rapee (1997) defienden que, en los niños con una vulnerabilidad genética a la ansiedad, el exceso de control o sobreprotección por parte de los padres puede reforzar dicha vulnerabilidad, reduciendo las oportunidades para desarrollar estrategias de afrontamiento, transmitiéndoles una percepción de inseguridad para conducir el entorno con éxito y aumentando la percepción de amenaza del entorno.

En definitiva, estos autores postulan que el exceso de control y la falta de afecto pueden hacer desarrollar a los niños una percepción de amenaza generalizada y un sentimiento de ineficacia personal o de incapacidad para afrontar los eventos amenazantes, siendo estos los principales componentes de la vulnerabilidad cognitiva 0 psicológica de los modelos cognitivos explicativos de la ansiedad. Según estos modelos, dicha vulnerabilidad interactúa con predisposiciones de personalidad y acontecimientos estresantes, explicando la aparición y desarrollo de la ansiedad (Chorpita y Barlow, 1998; Clark y Beck, 2012).

\section{Objetivo}

Con base en todo lo expuesto anteriormente, puesto que los estilos educativos son factores modificables sobre los cuales es posible actuar, y dados los altos índices de prevalencia, comorbilidad y las fuertes implicaciones de la ansiedad en la población infanto-juvenil, en esta revisión se pretende analizar la bibliografía existente para determinar si hay evidencia empírica consistente sobre la relación entre los estilos educativos 0 prácticas de crianza y la ansiedad infanto-juvenil. Concretamente se abordan dos cuestiones teóricas:

1) ¿Es consistente la evidencia empírica que sugiere que determinados estilos educativos se relacionan con la ansiedad en niños y adolescentes?

2) ¿Es congruente la evidencia empírica que indica que determinadas prácticas de crianza específicas se asocian con la ansiedad en la población infanto-juvenil?

\section{Metodología}

\subsection{Proceso de búsqueda}

Durante los meses de octubre y noviembre de 2014 se llevó a cabo un proceso de búsqueda bibliográfica sobre artículos que analizaran la relación de los estilos educativos 0 prácticas de crianza parentales con la ansiedad de niños y adolescentes. En una primera fase, se efectuó una búsqueda inicial para tener una aproximación del volumen de información e identificar los términos clave 0 descriptores, así como los criterios de selección de los artículos. En una segunda fase, se realizó una búsqueda bibliográfica sistemática en las siguientes bases de datos electrónicas: Dialnet, Redalyc, PsycInfo y Medline. Los términos clave o descriptores empleados fueron los siguientes: estilos educativos, prácticas de crianza, prácticas educati- 
vas, ansiedad, infantil, juvenil, infancia y adolescencia, para las bases de datos de lengua española (Dialnet y Redalyc) y parenting practices, parenting styles, anxiety, childhood y adolescence en las bases de datos de lengua inglesa (PyscInfo y Medline); los cuales fueron combinados de diversas formas para ampliar la búsqueda.

Estos descriptores produjeron un grupo inicial de artículos que fue reducido por etapas en función de los criterios de inclusión que se explicitan en el apartado siguiente. En una primera etapa se filtraron los artículos a partir del título y el resumen o abstract y, en una segunda, por el contenido del mismo. En total se seleccionó un artículo de la base de datos de Dialnet, cinco de Redalyc, quince artículos de PsycInfo y uno de Medline, comprendiendo un total de veintidós artículos que cumplían los criterios de inclusión.

\subsection{Selección de artículos: criterios de inclusión}

Los criterios de inclusión utilizados fueron los siguientes:

a) Artículos que incluyeran una medida directa y actual de los estilos educativos 0 de las prácticas de crianza de uno de los padres o de ambos.

b) Artículos que incluyeran una medida sobre la ansiedad de los niños, 0 bien estos contaran con el diagnóstico de, como mínimo, un trastorno de ansiedad.

c) Artículos en los que la asociación entre la ansiedad infanto-juvenil y las prácticas de crianza 0 estilos educativos parentales hubiera sido probada estadísticamente, y artículos que constituyeran revisiones bibliográficas. d) Artículos en los que la edad de los niños y adolescentes de la muestra no fuera superior a los 18 años.

e) Artículos cuya fecha de publicación fuera posterior al año 2000.

f) Artículos publicados en inglés, español o catalán y de los que se tuviera acceso completo al texto.

Fueron excluidos, por tanto, los artículos que asociaban la ansiedad infanto-juvenil exclusivamente con el tipo de apego 0 , únicamente, con aspectos del funcionamiento o tipología familiar, así como estudios retrospectivos, ya que no utilizan medidas directas y actuales de los estilos educativos 0 prácticas de crianza. Igualmente, se excluyeron los artículos que analizaban la relación de los estilos 0 prácticas de crianza con la ansiedad infanto-juvenil cuando esta fuera consecuencia de una enfermedad médica.

\subsection{Descripción de los artículos selec- cionados}

De los veintidós artículos seleccionados, tres de ellos (Bögels y Brechman-Toussaint, 2006; Cuervo, 2010; Wood et al., 2003) son revisiones bibliográficas y, dos más, estudios de metaanálisis (McLeod et al., 2006; Yap, Pilkington, Ryan y Jorm, 2014); el resto (diecisiete) son artículos de investigación. Respecto al diseño de investigación de los diecisiete estudios, dieciséis de ellos emplean un diseño transversal y el restante un diseño longitudinal (Muhtadie, Zhou, Eisenberg y Wang, 2013).

De estos diecisiete estudios, siete de ellos relacionan los estilos educativos 0 prácticas de crianza solo con la ansiedad (Erozkan, 2012; Hale, Engels y Meeus, 
2006; Lindhout, Markus, Hoogendijk y Boer, 2009; Luis et al., 2008; Varela, Niditch, Hensley-Maloney, Moore y Creveling, 2013; Varela, Sanchez-Sosa, Biggs y Luis, 2009; Woldfradt, Hempel y Miles, 2003); uno con la ansiedad y el trastorno de oposición desafiante (Hudson y Rapee, 2001); dos de ellos con problemas internalizantes (Laskey y Cartwright-Hatton, 2009; Muhtadie et al., 2013); y siete de estos con síntomas internalizantes y externalizantes por separado (Betancourt y Andrade, 2011; Franco, Pérez y de Dios, 2014; García-Linares, Cerezo, de la Torre, Carpio y Casanova, 2011; Nunes, Faraco, Vieira y Rubin, 2013; Oliva et al., 2007; Roelofs, Meesters, Teer-Huurne, Bamelis y Muris, 2006; Yahav, 2006).

Respecto a las medidas de los estilos educativos 0 prácticas de crianza, catorce de los artículos evalúan y analizan esta variable desde un enfoque dimensional (Betancourt y Andrade, 2011; Franco et al., 2014; García-Linares et al., 2011; Hale et al., 2006; Hudson y Rapee, 2001; Laskey y Cartwright-Hatton, 2009; Lindhout et al., 2009; Luis et al., 2008; Nunes et al., 2013; Oliva et al., 2007; Roelofs et al., 2006; Varela et al., 2013; Varela et al., 2009), dos artículos desde un enfoque tipológico (Erozkan, 2012; Muhtadie et al., 2013), y un único estudio analiza esta variable desde los dos enfoques existentes (Wolfradt et al., 2003).

\section{Resultados}

\subsection{Enfoque tipológico}

Por lo que respecta a los artículos que parten de un enfoque tipológico, solo se observa consistencia en los resultados del estilo autoritario. Este se ha relacionado con mayores niveles de ansiedad (sensibilidad a la ansiedad y ansiedad rasgo) en dos artículos (Erozkan, 2012; Wolfradt et al., 2003). En un tercer estudio (Muhtadie et al., 2013) se observa que la interacción del estilo autoritario con bajos niveles de control voluntario (temperamento) de los niños predice los problemas internalizantes. En la revisión bibliográfica de Cuervo (2010) también se señala la existencia de evidencias que relacionan este estilo con comportamientos internalizantes.

En esta línea, la investigación de Lindhout et al. (2009) demuestra que altos niveles de afecto negativo (o rechazo) y bajos niveles de promoción de la autonomía (polo opuesto al control), conjuntamente, se relacionan con la presencia de trastornos de ansiedad. La interacción de altos niveles de control parental, con bajos niveles de manifestaciones afectivas, coinciden con la definición del estilo autoritario; de modo que estos hallazgos son congruentes con los artículos que analizan el estilo autoritario y podrían sumarse a las evidencias que relacionan este estilo con mayores niveles de ansiedad.

Sin embargo, para el estilo democrático 0 autoritativo los resultados son más ambiguos, ya que, mientras que dos artículos (Erozkan, 2012; Muhtadie et al., 2013) demuestran que este estilo se relaciona con menores niveles de sensibilidad a la ansiedad 0 problemas internalizantes, en el tercero (Wolfradt et al., 2003) se observa que se asocia con niveles moderados de ansiedad rasgo. 
El resto de estilos educativos son diferentes en cada artículo, lo cual no permite establecer comparaciones entre los resultados.

\subsection{Enfoque dimensional}

Respecto a la dimensión afecto, observamos diferentes resultados dependiendo del origen geográfico de la muestra.

La mayoría de las investigaciones utilizadas para esta revisión ofrecen evidencias bastante consistentes sobre su relación con los niveles 0 síntomas de ansiedad 0 síntomas internalizantes en población europea generalmente. Más concretamente, el afecto 0 calidez se ha relacionado significativamente con menores niveles de ansiedad rasgo (Wolfradt et al., 2003) y síntomas internalizantes (Oliva et al., 2007); y el rechazo, como polo opuesto, se ha relacionado con mayores niveles o síntomas de ansiedad, o internalizantes, en siete investigaciones (Franco et al., 2014; García-Linares et al., 2011; Hale et al., 2006; Hudson y Rapee, 2001; Nunes et al., 2013; Roelofs et al., 2006; Yahav, 2006).

No obstante, se han encontrado incongruencias en los artículos que utilizan muestras de familias latinoamericanas que han emigrado a los Estados Unidos. El estudio de Luis et al. (2008) obtiene resultados en dirección contraria a los anteriores, esto es, halla que la falta de calidez y aceptación se asocia con menores niveles de ansiedad. En este estudio la muestra la componen niños y adolescentes de familias europeas y mejicanas residentes en Estados Unidos, y niños y adolescentes mejicanos que viven en Méjico. Sin embargo, en otro estudio (Varela et al.,
2009) que utiliza una muestra similar (niños y adolescentes de familias mejicanas residentes en Méjico, y niños de familias europeas y latinoamericanas que viven en Estados Unidos), los resultados indican que la aceptación del padre se asocia con menores síntomas de ansiedad, mientras que la de la madre se relaciona con mayores síntomas de ansiedad para los grupos de niños latinos y europeos residentes en Estados Unidos, pero no con el grupo de niños mejicanos que viven en su país de origen. La tercera investigación, cuya muestra la forman familias latinoamericanas que han emigrado a los Estados Unidos (Varela et al., 2013), no encuentra asociaciones entre esta dimensión y los síntomas de ansiedad infanto-juvenil. La inconsistencia de los resultados de los estudios, realizados con niños de familias latinoamericanas que viven en Estados Unidos, sugiere que es necesaria una mayor investigación para analizar las relaciones en este colectivo, ya que es posible que influyan características culturales, así como situacionales derivadas del proceso de migración 0 relacionadas con el contexto en el que viven estas familias, tales como posibles dificultades de acceso al trabajo, sanidad, educación, problemas económicos, asimilación cultural, etc. (Ramírez y de la Cruz, 2003; citado en Varela et al., 2009). Esto podría explicar la discrepancia con los resultados del resto de artículos (con muestras europeas generalmente), los cuales encuentran evidencias bastante consistentes sobre la relación entre el afecto 0 rechazo con la ansiedad infanto-juvenil.

Por otra parte, los estudios de metaanálisis analizados también demuestran 
que el rechazo se relaciona con mayores niveles de ansiedad. Así, en el estudio de Yap et al. (2014) se señala que la aversión se asocia con mayores niveles de ansiedad, con un tamaño del efecto medio; de manera similar, en el estudio de McLeod et al. (2006), se observa que la aversión se asocia con mayores niveles de ansiedad, y la calidez con menores niveles de ansiedad, pero con un tamaño del efecto pequeño, destacando que la aversión puede tener un gran impacto en la ansiedad más que la ausencia de crianza positiva.

Igualmente, las revisiones bibliográficas muestran evidencias de esta relación, pero indicando que la consistencia entre las investigaciones es menor que para la dimensión control en una de las revisiones (Bögels y Brechman-Toussaint, 2006), 0 solo resulta moderadamente consistente cuando se trata de estudios observacionales (Wood et al., 2003).

En cuanto a la dimensión control, los resultados de los artículos incluidos en esta revisión son menos consistentes, entre otros aspectos, porque las evidencias dependen de la conceptualización y la operativización del constructo empleado. Pese a esto, se han encontrado una serie de constructos que proporcionan hallazgos bastante consistentes.

Así, para el constructo control psicológico se observan evidencias generalmente consistentes a través de los estudios. Exceptuando la investigación de Nunes et al. (2013), que no encuentra correlaciones con la ansiedad, el resto de investigaciones (Betancourt y Andrade, 2011; Hale et al., 2006; Oliva et al., 2007; Wolfradt et al., 2003) muestran correlaciones positivas y significativas, indicando que dicho constructo se relaciona con mayores niveles 0 síntomas de ansiedad 0 síntomas internalizantes.

Asimismo, los estudios que analizan la sobreimplicación (Hale et al., 2006; Hudson y Rapee, 2001), el excesivo control (Luis et al., 2008) y el control hostil (Varela et al., 2009; Varela et al., 2013) también resultan bastante consistentes, demostrando que dichos constructos se relacionan con mayores niveles 0 síntomas de ansiedad 0 síntomas internalizantes. No obstante, cabría matizar que se manifiestan algunas diferencias, especialmente respecto al género de los progenitores y cuando intervienen factores culturales en dos de los artículos, tal y como se señala a continuación. En el estudio de Varela et al. (2009), mientras que el control de la madre se relaciona con los síntomas de ansiedad de los grupos de niños y adolescentes europeo-americanos y latinoamericanos, el control del padre solo se relaciona con los síntomas de ansiedad en el grupo de niños y adolescentes europeo-americanos. En el estudio de Luis et al. (2008), el control excesivo se asocia con mayores niveles de ansiedad para los niños y adolescentes mejicanos y europeo-americanos, pero con menores niveles de ansiedad para el grupo de niños y adolescentes mejicanos residentes en Estados Unidos.

Respecto al constructo disciplina rígida, dura o punitiva, los resultados coinciden en señalar su relación con mayores síntomas internalizantes (García-Linares et al., 2011; Laskey y Cartwright-Hatton, 2009), aunque con distinta magni- 
tud o fuerza de las asociaciones (siendo solo moderadas las asociaciones halladas en Laskey y Cartwright-Hatton, 2009), y con diferencias según el género del menor en uno de los estudios (en García Linares et al., 2011, las magnitudes de las correlaciones son menores en el caso de las chicas).

Por otro lado, para los constructos control conductual, sobreprotección y disciplina laxa (baja 0 indulgente), los resultados son menos congruentes y concluyentes. Para la sobreprotección paterna, en uno de los estudios (Yahav, 2006) no se hallan diferencias entre la sobreprotección de los padres de niños y adolescentes con síntomas internalizantes respecto a los del grupo control, pero sí en comparación con sus hermanos sin síntomas. En el otro estudio (Roelofs et al., 2006), se encuentran asociaciones positivas con los síntomas de ansiedad, pero la magnitud difiere según el género de los menores.

En relación con la disciplina laxa, baja 0 indulgente, uno de los estudios (Franco et al., 2014) obtiene que se relaciona con mayores niveles de retraimiento social y ansiedad, otro (García-Linares et al., 2011) halla asociaciones con los síntomas internalizantes pero solo para los chicos, y el tercero (Laskey y Cartwright-Hatton, 2009) no demuestra tales asociaciones.

Igualmente, las evidencias sobre el control conductual también son poco concluyentes, encontrando discrepancias en la magnitud de las asociaciones e incluso en la dirección de las mismas: Betancourt y Andrade (2011) obtienen que el control conductual materno correlaciona negativamente con todos los problemas emocionales, y el del padre solo con dos de los problemas (depresión y lesiones autoinfligidas). Y en el estudio de Oliva et al. (2007), las correlaciones halladas son bajas o poco significativas, incluso encontrando una relación positiva con los problemas internos cuando el control conductual es ejercido por la madre.

En relación con los estudios de metaanálisis analizados (McLeod et al., 2006; Yap et al., 2014), ambos demuestran que la sobreimplicación se relaciona con mayores niveles de ansiedad, pese a que los tamaños del efecto no son muy grandes.

Respecto a las revisiones bibliográficas, estas destacan la consistencia de las evidencias que relacionan el control con mayores niveles de ansiedad en los estudios observacionales. No obstante, cuando se trata de investigaciones que hacen uso de medidas de cuestionario, las conclusiones difieren en las dos revisiones: Bögels y Brechman-Toussaint (2006) afirman que existen evidencias consistentes sobre dicha relación, pero Wood et al. (2003) señalan que los resultados no son concluyentes.

\section{Discusión}

Respecto a la primera cuestión planteada para esta revisión, se puede concluir que el estilo autoritario se ha relacionado consistentemente con la ansiedad infanto-juvenil a lo largo de los estudios, asociándose a mayores niveles de ansiedad 0 síntomas internalizantes. Estos resultados concuerdan con la afirmación de Aroca et al. (2014, p. 217), quienes señalan que las familias que adoptan este estilo «suelen forzar a los menores a realizar ac- 
ciones que generan tensión y ansiedad en él». Asimismo, apoyan las evidencias que indican que este estilo provoca un impacto negativo sobre el ajuste y desarrollo del menor. El resto de estilos deben ser investigados con mayor profundidad.

En cuanto a la segunda cuestión, se han encontrado evidencias bastante consistentes que relacionan el afecto, la calidez y la aceptación con menores niveles 0 síntomas de ansiedad 0 síntomas internalizantes en población generalmente europea; y el rechazo, el control psicológico, la sobreimplicación, el excesivo control, la disciplina rígida y el control hostil, con mayores niveles 0 síntomas. El resto de constructos sobre la dimensión control no ofrecen datos suficientemente consistentes, ni permiten establecer conclusiones decisivas. Estos resultados coinciden con los planteamientos e hipótesis de los autores de los modelos cognitivos explicativos de la ansiedad, los cuales teorizan que el exceso de control y la falta de calidez pueden transmitir a los menores una percepción de amenaza generalizada y un sentimiento de ineficacia personal. Dichos componentes interactúan con predisposiciones de personalidad y acontecimientos estresantes, explicando la aparición y desarrollo de la ansiedad. Pese a que los resultados encajan con estos planteamientos, no constituyen pruebas empíricas de los mismos, esto es, no permiten demostrar tales hipótesis. Principalmente porque, aunque se analiza la relación entre estas prácticas y la ansiedad, no se examinan los mecanismos específicos que pueden mediar dicha relación (por ejemplo, los sesgos cognitivos de los menores: sesgo atencional hacia la información ame- nazante, interpretación de la información ambigua como amenazante, evaluaciones subestimadas de los propios recursos de afrontamiento, etc.), en los cuales se basan las hipótesis anteriores. Asimismo, la gran mayoría de las investigaciones analizadas en esta revisión no contemplan ni el temperamento ni la personalidad de los menores, siendo estos componentes también relevantes para la explicación de la ansiedad en las teorías cognitivas. Sería interesante examinar los mecanismos específicos que puedan mediar la relación entre los estilos educativos o prácticas de crianza con la ansiedad infanto-juvenil, así como analizar la interacción del temperamento de los menores con los estilos 0 prácticas educativas y la ansiedad, e incluso, examinar los posibles efectos interactivos de otros factores (como la ansiedad de los progenitores, las creencias y cogniciones sobre su propia competencia parental y sus propios hijos, el funcionamiento y tipología familiar, etc.) en futuras investigaciones.

Por otro lado, esta revisión ha permitido detectar una serie de limitaciones en el campo de la investigación actual que cabe señalar. Es posible que estas limitaciones puedan explicar, al menos en parte, la falta de congruencia de los resultados de algunos de los estudios. A su vez, plantean nuevas vías de indagación 0 cuestiones que deben ser abordadas en la investigación futura sobre la temática.

En primer lugar, en este trabajo se han agrupado los resultados del enfoque dimensional en función de dos dimensiones (afecto y control), pese a que no todas las investigaciones utilizan los mismos términos 0 constructos para evaluarlas. 
Además, cabe destacar que la mayoría de los estudios no definen las dimensiones 0 constructos evaluados. Esto resulta especialmente significativo en la dimensión control. Aunque en la dimensión afecto sí parece haber un mayor consenso entre los investigadores sobre su conceptualización, para la de control «los investigadores no se han puesto de acuerdo con respecto a los aspectos a incluir bajo esta etiqueta» (Oliva et al., 2007, p. 49), lo cual implica una «diversidad de criterios y resultados poco consistentes» (Aroca et al., 2014, p. 218), y de este modo, «resulta complicado saber cuál de ellas es la que realmente se relaciona con el ajuste de sus hijos e hijas» (Oliva et al., 2007, p. 49). Por tanto, se hace evidente la necesidad de definir con precisión las dimensiones evaluadas en las investigaciones, bajo unos mismos criterios y constructos.

Similarmente, respecto a la variable ansiedad, en esta revisión se han incluido estudios que utilizan diferentes constructos y medidas sobre esta problemática. Además, aunque la mayoría de los estudios utiliza muestras de niños 0 adolescentes no clínicos, tres de las investigaciones parten de los diagnósticos de algún trastorno de ansiedad de los menores. Es posible que haya variaciones de los efectos de las prácticas de crianza en los menores que padecen algún trastorno de ansiedad respecto a la población no clínica, o que el estado diagnóstico de los menores repercuta en las prácticas de crianza de los padres.

Igualmente, el hecho de incluir estudios que examinan la relación de los estilos o prácticas de crianza con los síntomas internalizantes, impide concluir que los hallazgos encontrados son específicos para la ansiedad. Gran parte de la literatura sobre esta temática se ha centrado en analizar la relación de los estilos educativos 0 prácticas de crianza con el ajuste interno del menor. De hecho, de las investigaciones seleccionadas, la mitad de estas examinan la relación con los problemas 0 síntomas internalizantes de forma conjunta.

Asimismo, pocas de las investigaciones revisadas, aparte de analizar la relación con la ansiedad, examinan la asociación con otras problemáticas, para comprobar si los resultados hallados son específicos para la ansiedad o comunes con otro tipo de problemáticas o para la psicopatología en general.

Consecuentemente, de cara a futuras investigaciones, sería conveniente examinar la relación de los estilos 0 prácticas de crianza con medidas de ansiedad en particular, e incluir grupos de niños 0 adolescentes con otras problemáticas, para comprobar si los resultados son específicos para la ansiedad 0 comunes con otras problemáticas.

En referencia a las características demográficas de las muestras de los estudios, cabe señalar, por un lado, que se han analizado los resultados de las investigaciones para la población infanto-juvenil sin diferenciar la etapa infantil de la adolescencia, puesto que gran parte de las investigaciones revisadas no realiza tal diferenciación. Es posible que los estilos 0 prácticas de crianza tengan un impacto diferente según la etapa del desarrollo del menor, o que los padres adapten sus estrategias 0 conductas según las características evolutivas de sus hijos. En esta línea, Yap et al. (2014) plantean que algu- 
nos de los factores que son especialmente relevantes en la adolescencia difieran, probablemente, de los que son más importantes en la infancia temprana. Por tanto, parece oportuno analizar la relación de forma diferencial para cada una de las etapas del desarrollo.

Por otro lado, aunque la gran mayoría de las investigaciones analizadas utilizan muestras de procedencia europea, también se incluyen estudios de otras culturas. Es probable que la cultura modere la relación entre los estilos educativos 0 prácticas de crianza y la ansiedad. De hecho, para la dimensión afecto, se han observado importantes discrepancias en los estudios con muestras de familias latinoamericanas que han emigrado a los Estados Unidos. Igualmente, para la dimensión control, también se han apuntado ciertas diferencias culturales. Algunos autores defienden que el control o las prácticas autoritarias parecen tener efectos neutrales -0 incluso positivos - en los niños latinoamericanos (Halgunseth, Ispa y Rudy, 2006; citado en Varela et al., 2009). Dichas prácticas son valoradas en las culturas colectivistas (como la latina y asiática), en tanto que son vistas como un mecanismo para inculcar el respeto hacia la autoridad y a las necesidades colectivas 0 familiares frente a las individuales (Luis et al., 2008; Varela et al., 2009); por tanto, pueden no tener las mismas repercusiones que en las culturas más individualistas como la europea. De este modo, estas discrepancias sugieren la necesidad de investigar la relación de los estilos 0 prácticas de crianza en diferentes grupos étnicos y culturales, y de examinar las posibles diferencias transculturales.
Respecto al género de los progenitores, la mayoría de las investigaciones han analizado los estilos o prácticas de crianza de ambos padres como unidad. A pesar de esto, los estudios que han considerado las prácticas de ambos padres por separado han encontrado diferencias en los resultados en función del género del progenitor. Esto podría estar indicando que la repercusión de las diferentes prácticas de crianza varía según el género de los progenitores, lo cual manifiesta la necesidad de considerar los estilos o prácticas de ambos padres por separado y analizar la implicación de esta variable en las investigaciones. Asimismo, sería interesante tener en cuenta la implicación del género de los menores en los resultados, ya que es posible que los niños y las niñas respondan diferencialmente a las prácticas de crianza (tal y como se ha apuntado en algunos estudios analizados en esta revisión, como el de García-Linares et al., 2011).

En cuanto a las medidas de evaluación, en esta revisión se han incluido investigaciones que han utilizado diferentes tipos de medidas. No obstante, excepto dos estudios, el resto ha empleado exclusivamente medidas de cuestionario para evaluar ambas variables, siendo medidas cuestionadas por varios autores. Por ejemplo, Holden y Edwards (1998; citado en McLeod et al., 2006) critican estas medidas por su falta de validez convergente. Sería conveniente, en futuras investigaciones, emplear diferentes medidas de evaluación, así como hacer uso de múltiples informantes.

Para concluir con las limitaciones, si bien la revisión ha aportado evidencias sobre la relación de determinados estilos 
0 dimensiones con la ansiedad, la naturaleza de dicha relación no se ha podido demostrar, ya que, exceptuando un estudio, el resto se tratan de investigaciones de diseño transversal. Por ello, no es posible demostrar hipótesis causales que expliquen una posible direccionalidad de los resultados obtenidos: si ciertos estilos 0 prácticas de crianza, junto con otros factores, pueden explicar la aparición y desarrollo de la ansiedad infanto-juvenil; si las propias características 0 estados internos de los menores determinan los estilos 0 prácticas de crianza; si la relación es bidireccional, etc. Por tanto, un reto para la investigación futura sería emplear diseños de investigación longitudinales, y de este modo esclarecer la naturaleza 0 dirección de los efectos.

En definitiva, en esta revisión se han encontrado evidencias generalmente consistentes que relacionan el afecto, la calidez y la aceptación con menores niveles 0 síntomas de ansiedad o síntomas internalizantes en población mayoritariamente europea; y el rechazo, el control psicológico, el excesivo control, la sobreimplicación, la disciplina rígida, el control hostil y el estilo autoritario con mayores niveles 0 síntomas de ansiedad 0 síntomas internalizantes. También se ha evidenciado la necesidad de continuar con el campo de investigación para resolver las limitaciones y abordar las nuevas cuestiones emergentes. Aunque los resultados no pueden considerarse totalmente definitivos y, a falta de corroborarlos con otras evidencias empíricas más consistentes, estos podrían tener importantes implicaciones en el campo práctico y profesional. Concretamente, en el desarrollo de programas pre- ventivos de educación familiar, centrados en orientar a los padres sobre el tipo de prácticas y conductas a desarrollar para evitar la aparición de problemas de ansiedad en sus hijos, y favorecer su desarrollo y bienestar; siempre, tratando de adaptar dichos programas a la singularidad de cada familia e individuo, y valorando la familia «como escenario de aprendizaje [a la vez que] medio educativo» (Torío, Peña y Rodríguez, 2008, p. 171). Igualmente, en el ámbito clínico, cabría incluir los estilos 0 prácticas de crianza parentales como elementos clave a trabajar en los programas de intervención o tratamiento de la ansiedad infanto-juvenil.

En conclusión, se hace evidente la necesidad de continuar la investigación en torno a esta temática y, de este modo, detectar los estilos o prácticas de crianza que constituyen factores de riesgo o protección para la ansiedad infanto-juvenil. Una vez identificados con una sólida evidencia empírica, el siguiente reto será desarrollar programas de prevención, e incluir dichos factores en el tratamiento e intervención de la ansiedad infanto-juvenil.

\section{Referencias bibliográficas}

Aroca, C., Cánovas, P., y Sahuquillo, P. (2014). Los estilos educativos. En P. Cánovas y P. M. Mateo (Coords.), Familias y menores: retos y propuestas pedagógicas (pp. 189-230). Valencia: Tirant Humanidades.

Betancourt, D., y Andrade, P. (2011). Control parental y problemas emocionales y de conducta en adolescentes. Revista Colombiana de Psicología, 20 (1), 27-24.

Bögels, S. M., y Brechman-Toussaint, M. L. (2006). Family issues in child anxiety: Attachment, 
family functioning, parental rearing and beliefs. Clinical Psychology Review, 26 (7), 834-856. doi: 10.1016/j.cpr.2005.08.001

Chorpita, B. F., y Barlow, D. H. (1998). The development of anxiety: the role of control in the early environment. Psychological Bulletin, 124 (1), 3-21. doi: 10.1037/0033-2909.124.1.3

Clark, D. A., y Beck, A. T. (2012). Terapia cognitiva para trastornos de ansiedad. Bilbao: Desclée de Brower.

Cuervo, A. (2010). Pautas de crianza y desarrollo socioafectivo en la infancia. Revista Diversitas: Perspectivas en Psicología, 6 (1), 111-121.

Dadds, M. R., y Barrett, P. M. (2001). Practitioner Review: Psychological management of anxiety disorders in childhood. Journal of Child Psychology and Psychiatry, 42 (8), 999-1011.

Darling, N., y Steinberg, L. (1993). Parenting style as context: an integrative model. Psychological Bulletin, 113 (3), 487-496. doi: 10.1037/00332909.113.3.487

Erozkan, A. (2012). Examination of relationship between anxiety sensitivity and parenting styles in adolescents. Educational Sciences: Theory \& Practice, 12 (1), 52-57.

Franco, N., Pérez, M. A., y De Dios, M. J. (2014). Relación entre los estilos de crianza parental y el desarrollo de ansiedad y conductas disruptivas en niños de 3 a 6 años. Revista de Psicología Clínica con Niños y Adolescentes, 1 (2), 149-156.

García-Linares, M. C., Cerezo, M. T., De la torre, M. J., Carpio, M. V., y Casanova, P. F. (2011). Prácticas educativas paternas y problemas internalizantes y externalizantes en adolescentes españoles. Psicothema, 23 (4), 654-659.

Hale, W. W. Engels, R. y Meeus, W. (2006). Adolescent's perceptions of parenting behaviours and its relationship to adolescent Generalized Anxiety Disorder symptoms. Journal of Adolescence, 29, 407-417. doi: 10.1016/j.adolescence.2005.08.002
Hudson, J. L., y Rapee, R. M. (2001). Parent-child interactions and anxiety disorders: an observational study. Behaviour Research and Therapy, 39, 1411-1427. doi: 10.1016/S00057967(00)00107-8

Laskey, B. J., y Cartwright-Hatton, S. (2009). Parental discipline behaviours and beliefs about their child: associations with child internalizing and mediation relationships. Child: care, health and development, 35 (5), 717-727. doi: 10.1111/j.1365-2214.2009.00977.x

Lindhout, I. E., Markus, M. TH., Hoogendijk, T. H. G., y Boer, F. (2009). Temperament and parental child-rearing style: unique contributions to clinical anxiety disorders in childhood. European Child \& Adolescent Psychiatry, 18 (7), 439-446. doi: 10.1007/s00787-009-0753-9

Luis, T. M., Varela, R. E., y Moore, K. W. (2008). Parenting practices and childhood anxiety in Mexican, Mexican American, and European American families. Journal of Anxiety Disorders, 22, 1011-1020. doi: 10.1016/j.janxdis.2007.11.001

McLeod, B. D., Wood, J. J., y Weisz, J. R. (2006). Examining the association between parenting and childhood anxiety: A meta-analysis. Clinical Psychology Review, 27 (2), 155-172. doi: 10.1016/j.cpr.2006.09.002

Muhtadie, L., Zhou, Q., Eisenberg, N., y Wang, Y. (2013). Predicting internalizing problems in Chinese children: The unique and interactive effects of parenting and child temperament. Development and Psychopathology, 25 (3), 653-667. doi: 10.1017/S0954579413000084

Nunes, S. A. N., Faraco, A. M. X., Vieira, M. L., y Rubin, K. H. (2013). Externalizing and internalizing problems: Contributions of attachment and parental practices. Reflexão e Crítica, 23 (3), 617-625. doi: 10.1590/S010279722013000300022

Oliva, A., y Parra, A. (2004). Contexto familiar y desarrollo psicológico durante la adolescencia. 
En E. Arranz (Ed.). Familia y desarrollo psicológico (pp. 96-123). Madrid: Pearson Prentice-Hall.

Oliva, A., Parra, A., Sánchez-Queija, I., y López, F. (2007). Estilos educativos materno y paterno: Evaluación y relación con el ajuste adolescente. Anales de Psicología, 23 (1), 49-56.

Rapee, R. M., (1997). Potential role of childrearing practices in the development of anxiety and depression. Clinical Psychology Review, 17 (1), 47-67.

Roelofs, J., Meesters, C., Teer-Huurne, M., Bamelis, L., y Muris, P. (2006). On the links between attachment style, parental rearing behaviours, and internalizing and externalizing problems in non-clinical children. Journal of Child and Family Studies, 15 (3), 331-344. doi: 10.1007/s10826-006-9025-1

Sala, J. (2002). Ideas previas sobre la docencia y competencias emocionales en estudiantes de Ciencias de la educación. revista española de pedagogía, 60 (223), 543-557.

Torío, S., Peña, J. V., y Rodríguez, Mª C. (2008). Estilos educativos parentales: revisión bibliográfica y reformulación teórica. Teoría de la Educación, 20, 151-178.

Varela, R. E., Niditch, L. A., Hensley-Maloney, L., Moore, K. W., y Creveling, C. C. (2013). Parenting Practices, Interpretive Biases, and Anxiety in Latino Children. Journal of Anxiety Disorders, 27, 171-177. doi: 10.1016/j.janxdis.2012.12.004
Varela, R. E., Sánchez-Sosa, J., Biggs, B. K., y Luis, T. M. (2009). Parenting strategies and socio-cultural influences in childhood anxiety: Mexican, Latin American descent, and European American families. Journal of Anxiety Disorders, 23, 609-616. doi: 10.1016/j.janxdis.2009.01.012

Wolfradt, U., Hempel, S., y Miles, J. N. V. (2003). Perceived parenting styles, depersonalisations, anxiety and coping behaviour in adolescents. Personality and Individual Differences, 34, 524-532. doi: 10.1016/S01918869(02)00092-2

Wood, J. J., McLeod, B. D., Sigman, M., Hwang, W. C., y Chu, B. C. (2003). Parenting and childhood anxiety: theory, empirical findings and future directions. Journal of Child Psychology and Psychiatry, 44 (1), 134-151. doi: 10.1111/1469-7610.00106

Yahav, R. (2006). The relationship between children's and adolescents' perceptions of parenting style and internal and external symptoms. Child, Care, Health and Development, 33 (4), 460-471. doi: $10.1111 / j .1365$ 2214.2006.00708.x

Yap, M. B. H., Pilkington, P. D., Ryan, S. M., y Jorm, A. F. (2014). Parental factors associated with depression and anxiety in young people: A systematic review and meta-analysis. Journal of Affective Disorders, 156, 8-23. doi: 10.1016/j.jad.2013.11.007 